\title{
EDITORIAL
}

\section{In This Issue: Changing Prescriptions for Change}

\author{
William R. Pbillips, MD, MPH, Editor
}

Ann Fam Med 2019;17:194-195. https://doi.org/10.1370/afm.2398.

$\mathrm{T}$ The prescription is often the most tangible evidence of care. Time and touch, listening and hearing, are more important, but prescriptions-for drugs, devices, and diagnostic tests-are more easily measured, monetized, and manipulated. In primary care practice, it is often the prescribing pen that produces the biggest costs or yields the greatest value. Likewise, in the design of our practices and health systems, prescriptive edicts now direct much of our work and clinical practice. For both the content and organization of patient care, research should inform the prescriptions we write and those we receive.

The opioid crisis threatens every part of our society. The lens of family medicine can help focus concern on our patients, families, and communities. In this issue, Clemans-Cope and her team from the Urban Institute document that over half a million US parents with opioid use disorder live with children in their homes. ${ }^{1}$ Millions more parents have other substance use disorders. Treatment and counseling rates are low for these parents, even for those with additional mental health problems. What more motivation do we need for detection, treatment, and systems of care than seeing patient lives ruined, patient lives lost, and generations of losses to come?

Even clinicians alert to the potential harms of these drugs report barriers to getting patients off chronic opioids. In a PBRN study, Tong and his team used mixed methods to study both patients and primary care clinicans. ${ }^{2}$ Only $1 \%$ of patients were on opioids for more than 3 months, and most came to the clinicians on preexisting prescriptions. Barriers to deprescribing chronic opioids included lack of time to manage chronic pain and lack of control over patients' other sources of opioids from hospitals or specialists.

The opioid crisis threatens all communities, from our inner cities to remote rural areas. Lin and Knudsen studied buprenorphine prescribers across the United States and report that in non-metro areas the burden of this care is met largely by primary care physicians, who more often work in small practices and accept Medicaid patients. ${ }^{3}$ Their buprenorphine management is similar to big-city prescribers.

Other prescription drugs present risks, too, particularly for fragile patients. Liew and colleagues from
Singapore performed a meta-analysis on potentially inappropriate prescribing in older adults in primary care. ${ }^{4}$ They found these prescriptions were associated with multiple adverse outcomes, including emergency room visits and hospitalizations, adverse drug events, functional decline, and health-related quality of life.

Injudicious use of antibiotics can present risks to patients and populations. Most antibiotics are prescribed for respiratory infections, most often in primary care settings. To help reduce child visits (and potentially unnecessary prescriptions) for respiratory infections, Schneider and her colleagues from the United Kingdom developed a novel online parent education resource that combined local microbiological surveillance data, symptom information, and homecare advice. ${ }^{5}$ They tested it with clinical vignettes in primary care and found it lowered mothers' intentions to bring their child in for a doctor visit.

Another strategy to optimize the use of antibiotics is to identify those patients at risk of serious complications. Moore and colleagues from Oxford studied over 500 UK practices and over 28,000 adult patients with lower respiratory tract infections to identify factors associated with serious adverse outcomes. ${ }^{6}$ Eight clinical risk factors fell into 3 major categories: symptom severity, patient vulnerability, and physiological impact.

Drugs and bugs are not the only threats to health. Health care systems - at least in the United Statescontinue to threaten health and health care. But, just how much return in improved health could we expect from increased investments in medical care? Kaplan and Milstein from Stanford used 4 methods to estimate the contributions of health care to life expectancy in the United States. ${ }^{7}$ They estimate that restricted access to medical care accounts for about $10 \%$ of premature death and adverse health outcomes. Patient behaviors and social determinants have much larger effects.

How much of the potential benefit of medical care is attributable to primary care? Would investment in primary care leverage potential returns in personal and population health? Answering those key questions will require improvements in our ability to measure the process and value of primary care. Etz and a multidisciplinary team used mixed methods to develop a new 
measure, concise but comprehensive, of the high-value aspects of primary care. ${ }^{8}$ Using novel crowd-sourced samples of patients, clinicans, and health care payors, they sought to systematically describe what provides value in primary care. Their new Person-Centered Primary Care Measure comprehends the broad scope of primary care in 11 domains: accessibility, comprehensiveness, continuity, integration, coordination, relationship, advocacy, family context, community context, health promotion, and goal-oriented care.

Dr Steven Woolf expands upon these concepts in an editorial to emphasize that medical care is necessary but not sufficient to improve population health and reduce health inequities. ${ }^{9}$ Social and system changes will be required to improve the health of our patients and communities, he argues from the point of view of an experienced family doctor and a population health leader.

Advances in science, best evidence, and prescriptions for practice can only help bring improvements in clinical care and patient outcomes if advances are broadly disseminated and effectively implemented. Miller, Rubenstein, Howard, and Crabtree offer a new theory for dissemination and implementation (D\&I) science that shifts assumptions to empower primary care practices. ${ }^{10}$ After a decade of limited success with practice-change initiatives that assume practices need external supports to meet external standards, these investigators have reimagined D\&I theory. They took lessons from innovative practices and used systems thinking, complexity theory, action research, and principles of community-based participatory research. They propose shifting the source and direction of change from outside-in to inside-out to empower practices to make fundamental, effective, and sustainable changes.

Also in this issue, authors shared innovations in their primary care practices. Mahoney and Ash from Stanford launched Humanwide, to provide patients with genetic screening, wearable sensors, health assessment, and wellness coaching. ${ }^{11}$ This synthesis of technologies for precision health in primary care aims to predict and prevent disease more effectively and to cure more precisely.

Reves and her team at Virginia Commonwealth University developed a 60 -second survey to identify patients' unmet social needs. ${ }^{12}$ Testing the 15 -item checklist in the emergency department and general medical hospital service, they found that over $60 \%$ of patients reported unmet social needs within the last month. Many patients reported multiple needs, most often transportation, food, and housing.

Ricketts, Nguyen, and Narasimhan at New York's Montefiore have developed a new smartphone app to screen pregnant and postpartum women for depression, substance use, and social needs. ${ }^{13}$ It also provides patients short articles about pregnancy, fetal development, and wellness and a bidirectional chat feature to encourage patient engagement.

We hope the articles in this issue-reviews, trials, proposals and innovations-challenge researchers, practitioners, and policy makers. Please share your comments and join our online discussion at http:// AnnFamMed.org.

\section{References}

1. Clemans-Cope L, Lynch V, Epstein M, Kenney GM. Opioid and substance use disorder and receipt of treatment among parents living with children in the United States, 2015-2017. Ann Fam Med. 2019; 17(3):207-211.

2. Tong ST, Hochheimer CJ, Brooks EM, et al. Chronic opioid prescribing in primary care: factors and perspectives. Ann Fam Med. 2019; 17(3):200-206.

3. Lin L, Knudsen HK. Comparing buprenorphine-prescribing physicians across nonmetropolitan and metropolitan areas in the United States. Ann Fam Med. 2019;17(3):212-220.

4. Liew TM, Lee CS, Goh SKL, Chang ZY. Potentially inappropriate prescribing among older persons: a meta-analysis of observational studies. Ann Fam Med. 2019;17(3):257-266.

5. Schneider A, Cabral C, Herd N, et al. Reducing primary care attendance intentions for pediatric respiratory tract infections. Ann Fam Med. 2019;17(3):239-249.

6. Moore M, Stuart B, Lown M, et al. Predictors of adverse outcomes in uncomplicated lower respiratory tract infections. Ann Fam Med. 2019;17(3):231-238.

7. Kaplan RM, Milstein A. Contributions of health care to longevity: a review of 4 estimation methods. Ann Fam Med. 2019;17(3):267-272.

8. Etz RS, Zyzanski SJ, Gonzalez MM, Reves SR, O’Neal JP, Stange KC. A new comprehensive measure of high-value aspects of primary care. Ann Fam Med. 2019;17(3):221-230.

9. Woolf SH. Necessary but not sufficient: why health care alone cannot improve population health and reduce health inequities. Ann Fam Med; 2019;17(3):196-199.

10. Miller WL, Rubinstein EB, Howard J, Crabtree BF. Shifting implementation science theory to empower primary care practices. Ann Fam Med. 2019;17(3):250-256.

11. Mahoney MR, Asch SM. Humanwide: a comprehensive data base for precision health in primary care. Ann Fam Med. 2019;17(3):273.

12. Reves SR, O'Neal J, Gonzalez MM, McHenry C, Favour M, Etz RS. A 60 -second survey to identify patients' unmet social needs. Ann Fam Med. 2019;17(3):274.

13. Ricketts S, Nguyen TNB, Narasimhan V. Screening for depression in pregnancy-there's an app for that! Ann Fam Med. 2019;17(3):275. 